University of Wollongong

Research Online

Faculty of Engineering and Information

Faculty of Engineering and Information

Sciences - Papers: Part B

Sciences

2018

\title{
Modeling supply network configuration problems with varying demand profiles
}

Subodha Dharmapriya

University of Wollongong, ssd889@uowmail.edu.au

Senevi Kiridena

University of Wollongong, skiriden@uow.edu.au

Nagesh Shukla

University of Technology, nshukla@uow.edu.au

Follow this and additional works at: https://ro.uow.edu.au/eispapers1

Part of the Engineering Commons, and the Science and Technology Studies Commons

Research Online is the open access institutional repository for the University of Wollongong. For further information contact the UOW Library: research-pubs@uow.edu.au 


\title{
Modeling supply network configuration problems with varying demand profiles
}

\author{
Abstract \\ In this paper, we develop a novel multi-objective modeling approach to support supply network \\ configuration decisions, while considering varying demand profiles. In so doing, we illustrate how such an \\ approach could contribute to building supply network robustness and resilience. The proposed model \\ entails two key objectives; minimizing lead time and cost across the supply network. The solution \\ approach first employs a bidding mechanism to select a set of supply network entities that match with a \\ given demand profile from a candidate pool of entities. It then applies the popular technique known as $\mathrm{N}$ \\ on-dominated Sorting Genetic Algorithm-II to generate a set of Pareto-optimal solutions representing \\ alternative supply network configurations. The proposed model is tested on a case study of a refrigerator \\ supply network to draw delivery time and cost comparisons under static and dynamic demand profiles.

\section{Disciplines} \\ Engineering | Science and Technology Studies

\section{Publication Details} \\ Dharmapriya, S., Kiridena, S. \& Shukla, N. (2018). Modeling supply network configuration problems with \\ varying demand profiles. 2018 IEEE Technology and Engineering Management Conference, TEMSCON \\ 2018 (pp. 1-6). United States: IEEE.
}




\section{Modeling supply network configuration problems with varying demand profiles}

\author{
Subodha Dharmapriya \\ School of Mechanical, Materials, \\ Mechatronic and Biomedical Engineering \\ Faculty of Engineering and Information \\ Sciences \\ University of Wollongong \\ Wollongong, Australia \\ ssd889@uowmail.edu.au
}

\author{
Senevi Kiridena \\ School of Mechanical, Materials, \\ Mechatronic and Biomedical Engineering \\ Faculty of Engineering and Information \\ Sciences \\ University of Wollongong \\ Wollongong, Australia \\ skiriden@uow.edu.au
}

\author{
Nagesh Shukla \\ School of Systems, Management and \\ Leadership \\ Faculty of Engineering and Information \\ Technology \\ University of Technology Sydney \\ Sydney, Australia \\ Nagesh.Shukla@uts.edu.au
}

\begin{abstract}
In this paper, we develop a novel multi-objective modeling approach to support supply network configuration decisions, while considering varying demand profiles. In so doing, we illustrate how such an approach could contribute to building supply network robustness and resilience. The proposed model entails two key objectives; minimizing lead time and cost across the supply network. The solution approach first employs a bidding mechanism to select a set of supply network entities that match with a given demand profile from a candidate pool of entities. It then applies the popular technique known as Nondominated Sorting Genetic Algorithm-II to generate a set of Pareto-optimal solutions representing alternative supply network configurations. The proposed model is tested on a case study of a refrigerator supply network to draw delivery time and cost comparisons under static and dynamic demand profiles.
\end{abstract}

Keywords-supply network configuration, demand profile, dynamics, bidding

\section{INTRODUCTION}

Facilitated by the ongoing advancements in technology and information systems, as well as the pursuit of broad-based initiatives such as Industry 4.0, supply networks are becoming more distributed and globally dispersed [1]. Additionally, shifting demand profiles and evolving competitive dynamics demand ongoing adjustments to supply chain (SC) structures [2]. The combined effects of these developments mean that appropriately responding to both unforeseen and anticipated disruptions is critical to maintaining fast, efficient and responsive SCs. The capacity for timely responding to these disruptions can be built through measures aimed at improving $\mathrm{SC}$ resilience and robustness as part of design considerations or operational control. Our review of literature on SC disruptions indicates that, compared to the work undertaken in the area of unforeseen disruptions, efforts directed towards the development of comprehensive responses to anticipated disruptions are sparse.

In this study, we develop a novel multi-objective modeling approach to support supply network configuration (SNC) decisions to suit varying demand profiles. In so doing, we make an effort to illustrate how SNC decisions could contribute to building SC robustness and resilience. The paper is organized as follows. Following this brief introduction, we present a summary review of extant literature on the SNC problem, while also focusing on measures aimed at addressing SC disruptions. Next, the mathematical formulation of the SNC problem is presented, followed by an overview of the solution approach employed in solving the SNC problem. We then test the proposed modeling approach using a case study of a refrigerator production network.

\section{LITERATURE REVIEW}

$\mathrm{SC}$ disruptions reported in the literature include unforeseen incidents such as transportation mishaps, natural calamities, and intentional attacks [3], as well as anticipated circumstances like facility breakdowns, failures of the supplier base, offensive actions of competitors and abrupt changes in demand [4-5]. Some authors have treated such events in terms of endogenous disruptions and exogenous disruptions [6]. Irrespective of the way they are classified, all disruptions are known to induce significant risks in terms of their impact on SC functioning or performance. Mitigating the impact of such risks involves improving SC robustness and resilience through building capacity to: withstand disruptions, including any structural adjustments required; respond quickly to disruptions; and recover effectively from any disruptive incidents [7-8]. Specific measures of risk mitigation that have been proposed in the literature include: strategies such as holding buffer stocks, maintaining back-up capacity, multiple-sourcing and adaptive ordering [3-4]; structural adjustments in supply networks [2]; and dynamic integration of logistics capabilities [9]. For instance, [10] proposed six measures covering both operational strategies and structural adjustments to deal with SC disruptions and uncertainty, namely facility dispersion, facility reinforcement, use of sub-assemblies, multiple sourcing, and keeping inventory and considering primary and alternative bills of material (BOM). Overall, the literature cited above highlights the existence of an array of measures that could potentially be used to mitigate the risks associated with $\mathrm{SC}$ disruptions, through building $\mathrm{SC}$ robustness and resilience. Such capacity can be built through SNC decisions leading to targeted proactive and reactive actions.

In general, the term $\mathrm{SC}$ refers to the sequential arrangement of organizational entities involved in acquiring raw materials, transforming them into components and assemblies, and then distributing the final product to end-users. However, given that an organizational entity can be part of more than one SC, a 
more realistic alternative would be to consider the notion of supply network (SN). With the notion of $\mathrm{SN}$ comes the existence of multiple SCs that are capable of catering to a given demand profile. This then introduces the possibility of differentiating such SCs with respect to their performance in terms of speed, efficiency and responsiveness. It is common practice in the industry to use the same set of supply entities for a given demand profile and for a prolonged period, considering such factors as the benefits of maintaining long-term relationships, contractual arrangements and ease of coordination and communication [11]. However, on the one hand, sticking to the same SC for too long can lead to the loss of competitiveness at the SC level, because developments such as the emergence of high-performing SC entities, the adoption of new technology and the introduction of new and better substitute products can significantly alter the overall competitiveness of alternative SCs. On the other hand, shifting demand profiles means a SC that has been configured to serve a given demand profile at a particular point in time could become less competitive if it is no longer aligned with the current demand profile [12-13]. As such, SNC decisions can play a critical role in sustaining the overall SC performance under evolving conditions or in the face of disruptions.

SNCs are alternative arrangements of organizational entities, processes and resources where, as a whole, they can be differentiated based on key performance metrics such as cost, lead-time and fill rate [14-16]. Previous studies have addressed $\mathrm{SN}$ design issues related to the decisions involving facility location, supplier selection and the optimal number of facilities required, often assuming static conditions and centralized decision-making [17-18]. However, more recent literature has contributed to accounting for disruptions and uncertainties in the design stage by introducing stochastic programing to the modeling environment [19]. There are also several other studies that have attempted to address the structural, spatial and temporal aspects of SNC decisions with the help of simulation modeling approaches, including the application of metaheuristics [20]. While structural characteristics are substantially covered in the literature, spatial and temporal characteristics such as changing demand profiles need to be further explored [20]. Although a market segment could be profiled using multiple attributes such as product volume, delivery time and price range, the treatment of demand profiles in existing SNC literature limits to the attribute of volume [20]. Similarly, changes in demand profile over time have not adequately addressed in the literature [20]. As such, this study focuses on dynamic demand profiles defined by the attributes of product volume, expected delivery time and willingness to pay (WTP) price.

Similar to the areas of research such as SN design and logistics network planning, optimization techniques play a major role in SNC literature. In most cases, SNC problems are considered to be of combinatorial optimization type and, therefore, researchers have used meta-heuristics approaches. Among them, the Genetic Algorithm [15-16], the Ant Colony Optimization [21] and the Bee Algorithm [22] are widely used for SNC. The Ant Colony Optimization and Bee Algorithmbased studies address multiple objectives such as optimizing both delivery time and cost. Agent-based modeling has also been used in a few studies [23-25], often, implemented in the context of rather narrowly defined SNs (e.g. single product and static demand). Reference [23] combined both agent-based modeling and optimization.

Overall, the literature points to several limitations in the current approaches to modeling structural, spatial and temporal characteristics of SNs. This study covers these three aspects with a focus on modeling multi-echelon SNs consisting of geographically dispersed entities responding to varying demand profiles. The proposed model entails two objectives; minimizing delivery time (DT) and total supply network cost (TSNC). The solution methodology involves a bidding mechanism to select SN entities from an available pool, and Non-dominated Sorting Genetic Algorithm-II NSGA-II is used to find a set of Pareto optimal solutions which meet the two key objectives referred to above. This model is tested on a case study of a refrigerator $\mathrm{SN}$.

\section{MATHEMATICAL FORMULATION}

In this study, we consider a $\mathrm{SN}$ with $I$ number of stages. These stages could be any one of the three types, sourcing, manufacturing or distribution, and are represented by a set $\mathbf{S}=$ $\left(\mathbf{S}_{1} \ldots \mathbf{S}_{\mathrm{i}} \ldots \mathbf{S}_{\mathrm{I}}\right)$, where, $\mathbf{S}_{i}$ represents $i^{\text {th }}$ stage of the SN. At each stage, there could be several types of raw material, product component or sub-assembly involved, depending on the bill of material (BOM) representing a given product. Each of these raw material, component and sub-assembly types is represented as a node in the SN. Accordingly, there could be multiple nodes at any stage. If there is a total of $J$ nodes in the $\mathrm{SN}$, then the nodes in the $i^{\text {th }}$ stage of the $\mathrm{SN}$ are represented by the set $\mathbf{S}_{\mathrm{i}}=\left(\mathbf{N}_{\mathrm{im}} \ldots \mathbf{N}_{\mathrm{ij}} \ldots \mathbf{N}_{\mathrm{in}}\right)$, where, $\mathrm{j} \in(\mathrm{m}, \mathrm{m}+1, \mathrm{~m}+2, \ldots, \mathrm{n})$. At each node, we consider that there are multiple competing supply entities, termed as entity options, that are capable of performing the value-adding functions at the respective node. Depending on such factors as location of facilities, capacity of their plants and the processes or technologies utilized, these

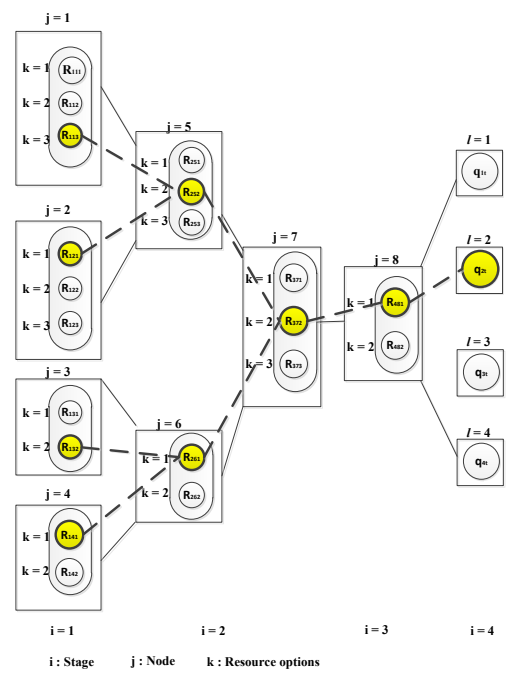

Fig. 1: Conceptual representation of a SN (with the selected SNC highlighted) 
entities can compete with each other on cost, lead time or quality parameters. For example, a local supply entity may be able to supply a component at a higher price with a shorter lead-time, whereas an overseas supplier may be able to supply it at a much lower price but with a considerably longer leadtime. At a given node $\mathbf{N}_{\mathrm{ij}}$, a set of entity options is given by $\mathbf{N}_{\mathrm{ij}}$ $=\left\{\mathrm{R}_{\mathrm{ij} 1 \ldots} \mathrm{R}_{\mathrm{ijk}} \ldots \mathrm{R}_{\mathrm{ijp}}\right\}$ where $R_{i j k}$ is the $k^{\text {th }}$ entity option at node $j$ of stage $i$. Here, $p$ is the maximum number of entity options available at $\mathbf{N}_{\mathrm{ij}}$. Fig. 1 illustrates this $\mathrm{SN}$ graphically with multiple entity options available at each node of a given stage. The SN marked in dashed lines is an instantiation of the SN identified as feasible for fulfilling a given demand profile. In this instance, entity options selected in stage 1 are $R_{113}$ and $R_{121}$, in stage 2 are $R_{252}$ and $R_{261}$, in stage 3 is $R_{372}$ and in stage 4 is $R_{481}$, so as to fulfil the order profile of consumer region 2.

The objective of the SNC problem is to fulfil the demand profile specific requirements such as product volume and due date at the minimal SC-wide cost and delivery lead time. As such, this problem is of combinatorial optimization type where the optimal set of entity options at each stage are selected for a specific demand profile.

The SNC problem is mathematically expressed as follows. Equation 1 and 2 represent objectives of the problem which are to minimize the total SN cost (TSNC) and delivery time (DT) by optimally configuring the $\mathrm{SN}$ to suit a given demand profile. The TSNC consists of two primary elements, namely the processing cost (PC) and transportation cost (TC), as represented by the first and second terms of (1), respectively. Similarly, DT includes the processing time (PT) and transportation time (TT), as represented by the first and second terms of (2), respectively. Unit processing cost of entity option $R_{i j k}$ to produce an item is denoted by $C_{i j k}$ and related processing time is taken as $P T_{i j k}$. In this case, we have considered transportation cost to be proportionate to the distance between supply entities. The distance between two selected entity options ( $R_{i j k}$ and $R_{i^{\prime}{ }^{\prime} k^{\prime}}$ ) at two consecutive stages ( $i$ and $i^{\prime}$ ) is indicated by $D_{i j k \rightarrow i^{\prime} j} k^{\prime}$ ' and unit distance transportation cost is

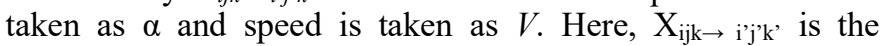
decision variable which has value 1 when option $R_{i j k}$ in stage $i$ and option $R_{i^{\prime} j^{\prime} k}$ ' in stage $(i+1)$ (i.e. $i^{\prime}$ ) are selected to fulfil a given order; otherwise it is 0 . The aggregate demand (volume) in consumer region $l$ at time $t$ is represented as $Q_{l t}$. We assume that $Q_{l t}$ follows the normal distribution $N\left(\mu_{q l}, \sigma_{q l}\right)$. The demand for each item at other upstream stages of the $\mathrm{SN}$ is determined by taking into account both demand at the final stage and the BOM of the product. If $\delta_{i j}$ represents the number of components per product required at node $\mathbf{N}_{\mathrm{ij}}$ according to the BOM, then demand of that node $\mathbf{N}_{\mathrm{ij}}$ is calculated as $\delta_{i j}$. $Q_{l t}$.

The objective function of this SNC problem is subject to several constraints. Equation (3) sets that only one entity option is selected at each node to satisfy the given demand profile. Equation (4) ensures that a potential entity option should have the production capacity to supply the required number of units $\left(\delta_{i j} . Q_{l t}\right)$ to satisfy the demand $Q_{l t}$. The available production

\section{Minimize the TSNC:}

$$
\begin{gathered}
\operatorname{Minimise}\left(F_{1}\right)=\sum_{t \in T} \sum_{l \in L} \sum_{S_{i} \in S} \sum_{N_{i j} \in S_{i}} \sum_{R_{i j k} \in N_{i j}} y_{i j k} \cdot C_{i j k} \cdot \delta_{i j} \cdot q_{l t}+ \\
\sum_{S_{i} \in S} \sum_{N_{i j} \in S_{i}} \sum_{R_{i j k} \in N_{i j}} \sum_{S_{i} \in S} \sum_{N_{i j} \in S_{i}} \sum_{R_{i^{\prime} j^{\prime}} k^{\prime} \in N_{i^{\prime} j^{\prime}}} x_{i j k \rightarrow i^{\prime} j^{\prime} k^{\prime}} \cdot \alpha \cdot D_{i j k \rightarrow i^{\prime} j^{\prime} k^{\prime}}
\end{gathered}
$$

Minimize DT:

$$
\begin{aligned}
& \operatorname{Minimise}\left(F_{2}\right)=\sum \sum_{t \in T} \sum_{l \in L} \sum_{S_{i} \in S} \sum_{N_{i j} \in S_{i} R_{i j k} \in N_{i j}} y_{i j k} \cdot P T_{i j k t} \cdot \delta_{i j} \cdot q_{l t}+ \\
& \sum_{S_{i} \in S} \sum_{N_{i j} \in S_{i}} \sum_{R_{i j k} \in N_{i j}} \sum_{S_{i} \in S} \sum_{N_{i j} \in S_{i}} \sum_{R_{i^{\prime} j^{\prime} k^{\prime}} \in N_{i^{\prime} j^{\prime}}} x_{i j k \rightarrow i^{\prime} j^{\prime} k^{\prime}} \cdot D_{i j k \rightarrow i^{\prime} j^{\prime} k^{\prime}} / V
\end{aligned}
$$

Subject to :

Entity option selection constraint:

$$
\sum_{k \in K_{j}} y_{i j k}=1
$$

Production capacity constraint:

$$
P C_{i j k l}^{\text {daily }}(T-t) \geq \delta_{i j} \cdot Q_{l t}
$$

Bidding cost evaluating constraint:

$$
b_{i j}^{c} \sim r n d\left[N_{i j}^{P C} \cdot p_{l t}, 0.85 N_{i j}^{P C} \cdot p_{l t}\right] \text { and } C_{i j k} \cdot y_{i j k} \leq b_{i j}^{c}
$$

Bidding time evaluating constraint:

$$
\begin{aligned}
& b_{i j}^{p t} \sim \operatorname{rnd}\left[N_{i j}^{D T} \cdot D T_{l t}, 0.85 . N_{i j}^{D T} . D T_{l t}\right] \text { and } P T_{i j k} \cdot y_{i j k} \leq b_{i j}^{p t} \\
& \forall i \in I ; i^{\prime}=i+1 ; j, j^{\prime} \in J ; k \in K_{j} ; k^{\prime} \in K_{j}^{\prime} ; t \in T ; l \in L
\end{aligned}
$$

capacity is calculated as $P C_{i j k l}^{\text {daily }}(T-t)$ where $\mathrm{T}$ is the end of the review period, and $\mathrm{t}$ is the start date for fulfilling the demand profile of the consumer region $l$. Equation (5) and (6) ensures that entity option $R_{i j k}$ gets selected if the $C_{i j k}$ of $R_{i j k}$ is below the reference cost $\left(b_{i j}^{c}\right)$ and $P T_{i j k}$ is less than the reference processing time ${ }^{\left(b_{i j}^{p t}\right)}$. Both $b_{i j}^{c}$ and $b_{i j}^{p t}$ are random numbers generated to fit within the upper and lower bounds of the estimated processing cost and processing time of the corresponding node, respectively. The upper and lower bounds for $b_{i j}^{c}$ are $N_{i j}^{P C} . P_{l t}$ and $0.85 N_{i j}^{P C} . P_{l t}$ where $N_{i j}^{P C}$ is a pre-defined processing cost proportion of $\mathbf{N}_{\mathrm{ij}}$ and $P_{l t}$ is WTP price of the given consumer region $l$. The upper and lower bounds of $b_{i j}^{p t}$ for processing time are $N_{i j}^{P T} \cdot D T_{l t}$ and $0.85 . N_{i j}^{P T} \cdot D T_{l t}$ where $N_{i j}^{P T}$ is the processing time proportion assigned to $\mathbf{N}_{\mathrm{ij}}$ and $D T_{l t}$ is expected delivery time for region $l$.

\section{SOLUTION APPROACH}

The methodological approach followed in solving the SNC problem is illustrated in Fig. 2. Initially, the demand profile of each consumer region is estimated in terms of volume $\left(\mathrm{Q}_{\mathrm{lt}}\right)$, WTP price $\left(\mathrm{P}_{1 \mathrm{t}}\right)$ and expected delivery time $\left(\mathrm{DT}_{\mathrm{lt}}\right)$. 
Then, at the order processing stage, $\mathrm{Q}_{\mathrm{It}}$ of the demand profile is processed using BOM to determine the required number of units $\left(\delta_{\mathrm{ij}}\right.$. $\left.\mathrm{Q}_{\mathrm{It}}\right)$ from each node $\mathbf{N}_{\mathrm{ij}}$. This is informed to supply entities through the supply entity selection stage. The bidding process considers $P_{l t}$ and $D T_{l t}$ to calculate the maximum acceptable price $\left(N_{i j}^{P C} . P_{l t}\right)$ and processing time $\left(N_{i j}^{P T} . D T_{l t}\right)$ at each node. This information is then made available to be used at the entity option selection step. At this step a bidding mechanism is employed to select a set of feasible entities from among the candidate entity options at each node. Accordingly, $R_{i j k}$ at each node are informed of the number of units required. Bidding mechanism initiates with generating $b_{i j}^{c}$ and $b_{i j}^{p t}$ as given in (5) and (6). As mentioned in section III, $b_{i j}^{c}$ is a random number that lies in the range of ${ }^{P C}$ ij.$P_{l t}$ and $0.85 N_{i j}^{P C} \cdot P_{l t}$. For example, if $N_{i j}^{P C}$ is equivalent to $20 \%$ of WTP price (i.e. $0.2 \mathrm{P}_{\mathrm{lt}}$ ), then $b_{i j}^{c}$ lies in the range of lower bound (i.e. $85 \%$ of $0.2 \mathrm{P}_{\mathrm{tt}}$ ) and upper bound (i.e. $0.2 \mathrm{P}_{\mathrm{lt}}$ ). Once the corresponding bids (i.e. $\mathrm{C}_{\mathrm{ijk}}$ and $\mathrm{PT}_{\mathrm{ijk}}$ ) to supply the required number of units are received from each $R_{i j k}$ at each node, these bids are then compared with the $b_{i j}^{c}$ and $b_{i j}^{p t}$. Bids which are lower than both $b_{i j}^{c}$ and $b_{i j}^{p t}$ are considered as winning bids; i.e. candidates $R_{i j k}$ are sent to the optimization step.

NSGA-II (as outlined in Fig. 3), one of the most popular multi-objective optimization algorithms has been used to find the optimal set of SNCs [26]. NSGA-II starts with initial population (i.e. parents), which is the set of SNCs having one entity option from each node. Then, the TSNC $\left(\mathrm{F}_{1}\right)$ and DT $\left(\mathrm{F}_{2}\right)$ are calculated to rank the population using the sorting algorithm known as Pareto-fast non-dominated (PF-ND). In the process of determining TSNC and DT, transporters are contacted to get the relevant transportation time and cost between entity options. Then, the standard genetic operators are applied (i.e. selection, crossover and mutation) within the defined criteria for ceasing the process of iterations. Elitism is achieved by combining the chosen attributes of parents and children, and are ranked with the use of PR-ND sorting. The next generation is selected from the highest ranking population, which is then sent to the following generation. This process continues until the ceasing criteria are met. Finally, the solutions in the Pareto front are taken as the optimal SNCs for a given set of demand profile requirements.

\section{CASE STUDY ANALYSIS}

In this study, we adapted a dataset pertaining to refrigerator

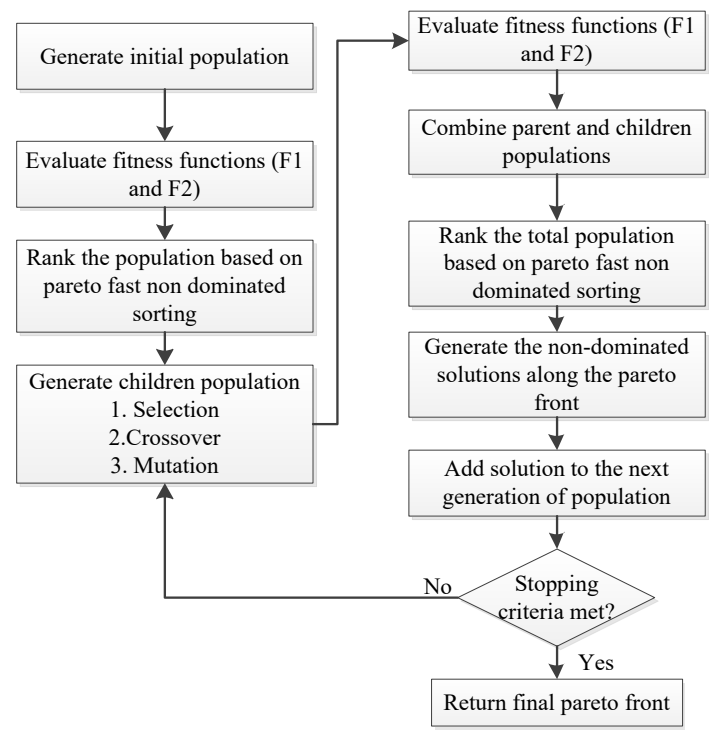

Fig. 2. Steps in the NSGA-II algorithm

production which was initially used by [27], and later modified by [28-29] with added parameters related to logistics networks, to optimize the lifecycle cost. To help demonstrate the efficacy of the proposed model, we introduced several new parameters to suit the specific SNC problem context. Further details about these parameters and associated data are presented below.

There are five stages in the refrigerator SN; three supply stages (i.e. raw material, component and module), the final assembly stage and the distribution (of finished products) stage. There are 25 different components manufactured using four different types of raw material, namely Iron (Fe), Plastic, Aluminium (Al) and Copper $(\mathrm{Cu})$. Based on the type of raw material used and the nature of the manufacturing process, we categorized 25 components into five groups, aimed at reducing the complexity of the SN. In the original data set, there were 13 modules, some of which were sub-modules of the others. We classified these 13 modules into two main modules, which are assembled to form the final product. The final products are sent to distribution centers through which retailers at each consumer region receive goods. Accordingly, there is more than one node (i.e. $\mathbf{N}_{\mathrm{ij}}$ ) in each stage and there are multiple entity options (i.e. $\mathrm{R}_{\mathrm{ijk}}$ ) available to perform the required value-adding functions at each node. The connectivity between the nodes is shown in Fig. 4. We used ten consumer regions in Europe with distinct demand profiles considering the differences in price level index and individual income. The possible variations in the

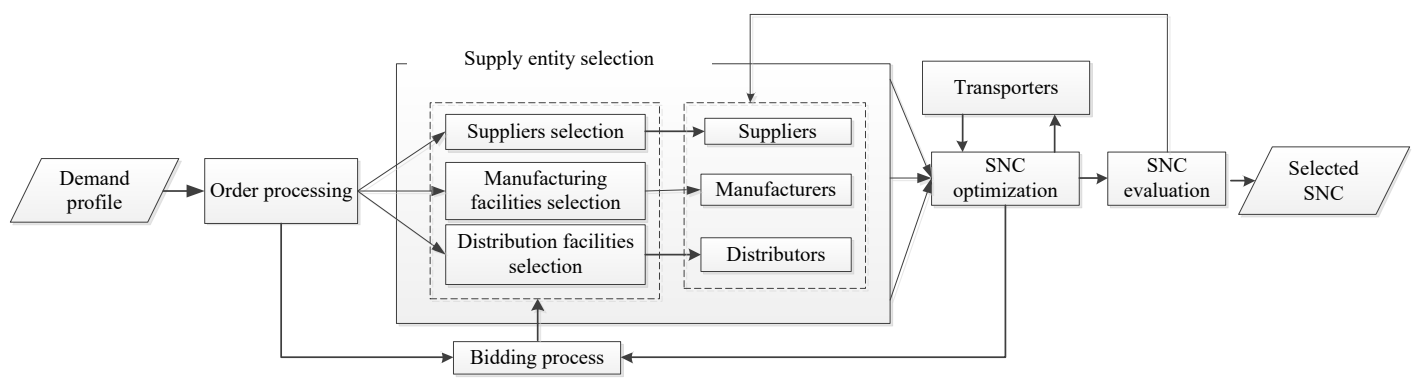

Fig. 3. Overview of the solution approach used 


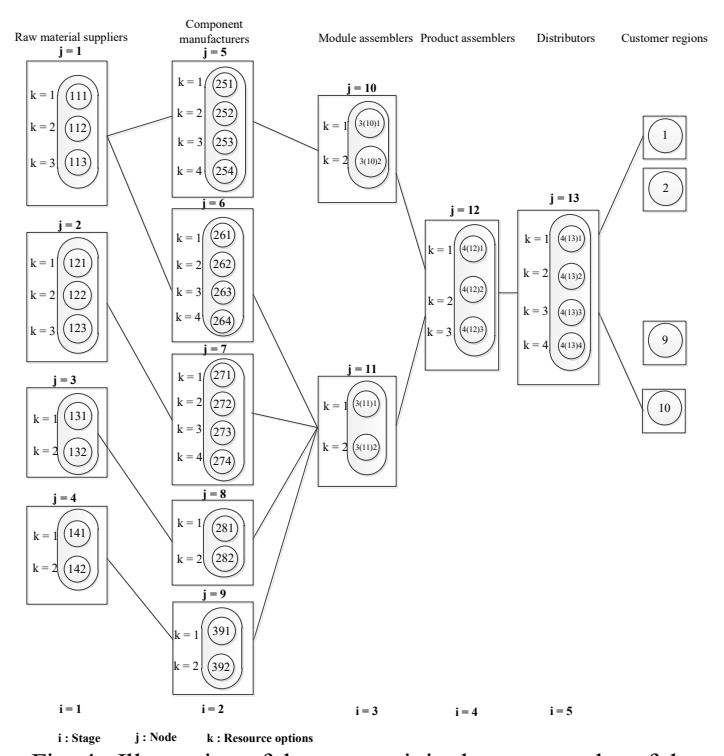

Fig. 4. Illustration of the connectivity between nodes of the refrigerator production network

TABLE 1. DEMAND Profile OF EACH CONSUMER REGION

\begin{tabular}{|l|l|l|l|}
\hline Cus. ID & $\begin{array}{c}\text { Volume } \\
(\text { units })\left(\boldsymbol{\mu}_{\mathrm{Q} 1}, \boldsymbol{\sigma}_{\mathrm{QI}}\right)\end{array}$ & $\begin{array}{c}\text { Delivery time } \\
(\text { days })\left(\boldsymbol{\mu}_{\mathrm{DT}}, \boldsymbol{\sigma}_{\mathrm{DTI}}\right)\end{array}$ & $\begin{array}{c}\text { WTP cost } \\
(\text { dollars })\left(\boldsymbol{\mu}_{\mathrm{Pl}}, \boldsymbol{\sigma}_{\mathrm{PI}}\right)\end{array}$ \\
\hline 1 & $(33,10)$ & $(30,10)$ & $(1000,150)$ \\
\hline 2 & $(40,20)$ & $(15,10)$ & $(900,100)$ \\
\hline 3 & $(100,20)$ & $(55,25)$ & $(1300,150)$ \\
\hline 4 & $(100,50)$ & $(40,10)$ & $(1100,100)$ \\
\hline 5 & $(40,14)$ & $(40,10)$ & $(1500,80)$ \\
\hline 6 & $(80,10)$ & $(55,15)$ & $(1200,150)$ \\
\hline 7 & $(120,16)$ & $(65,10)$ & $(1500,170)$ \\
\hline 8 & $(60,10)$ & $(45,7)$ & $(1100,80)$ \\
\hline 9 & $(150,12)$ & $(85,13)$ & $(1200,150)$ \\
\hline 10 & $(50,15)$ & $(40,7)$ & $(800,150)$ \\
\hline
\end{tabular}

TABle II. SOlutions For StATIC DEMAND Profiles (EXPERIMENT 1)

\begin{tabular}{|l|l|l|c|c|c|c|}
\hline \multicolumn{3}{|c|}{ Experiment 1 setting } & \multicolumn{2}{|c|}{ SNC solutions } & \multicolumn{2}{c|}{ \% Saving } \\
\hline $\begin{array}{c}\text { Cus. } \\
\text { ID }\end{array}$ & $\begin{array}{c}\text { Order } \\
\text { arrival } \\
\text { day }\end{array}$ & $\begin{array}{c}\text { Demand profile } \\
\left(\boldsymbol{Q}_{l,} \text { DT } \boldsymbol{T}_{l,} \boldsymbol{P}_{l t}\right)\end{array}$ & $\begin{array}{c}\text { TSNC } \\
\text { range }\end{array}$ & $\begin{array}{c}\text { DT } \\
\text { range }\end{array}$ & \multicolumn{1}{c|}{ Cost } & Time \\
\hline 1 & 1 & $(33,30,1000)$ & {$[717,746]$} & {$[17,21]$} & {$[25,28]$} & {$[30,43]$} \\
\hline 2 & 10 & $(40,15,900)$ & {$[710,786]$} & {$[20,23]$} & {$[13,21]$} & $-[33,53]$ \\
\hline 3 & 20 & $(100,55,1300)$ & {$[696,788]$} & {$[38,46]$} & {$[39,46]$} & {$[31,16]$} \\
\hline 4 & 45 & $(100,40,1100)$ & {$[739,802]$} & {$[29,35]$} & {$[27,33]$} & {$[12,27]$} \\
\hline 5 & 60 & $(40,40,1500)$ & {$[711,758]$} & {$[19,24]$} & {$[49,53]$} & {$[53,40]$} \\
\hline 6 & 75 & $(80,55,1200)$ & {$[698,793]$} & {$[31,39]$} & {$[34,42]$} & {$[44,29]$} \\
\hline 7 & 80 & $(120,65,1500)$ & {$[765,791]$} & {$[45,55]$} & {$[47,49]$} & {$[44,15]$} \\
\hline 8 & 85 & $(60,45,1100)$ & {$[703,799]$} & {$[26,33]$} & {$[27,36]$} & {$[42,27]$} \\
\hline 9 & 90 & $(150,85,1200)$ & {$[692,777]$} & {$[56,67]$} & {$[35,42]$} & {$[30,16]$} \\
\hline 10 & 120 & $(50,40,700)$ & {$[704,737]$} & {$[22,26]$} & $-[0.5,5.2]$ & {$[45,35]$} \\
\hline
\end{tabular}

attributes of the demand profile as defined by their mean and standard deviation are given in Table I.

Two experiments were carried out. In experiment 1 , we considered a static demand profile by employing only the mean value of each demand attribute of the respective demand profile. In experiment 2, dynamic demand profiles were
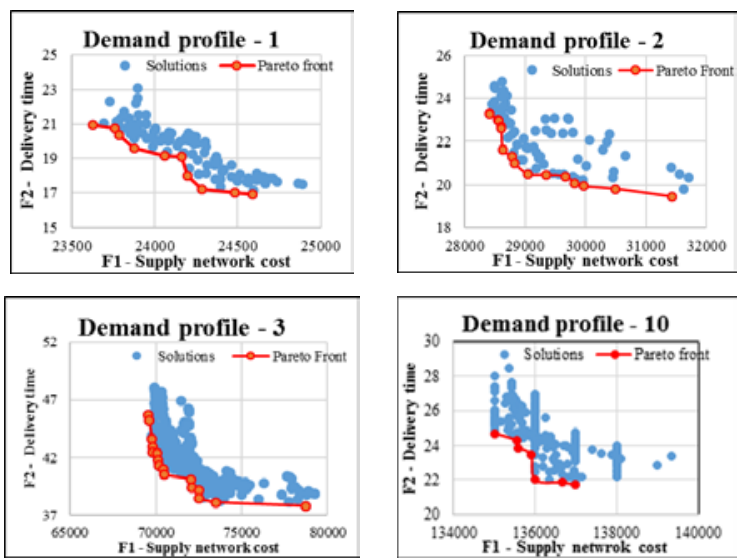

Fig. 5. Pareto optimal solutions (representing non-dominated SNC alternatives) generated by NSGA-II (in 100 runs)

TABLE III. SOLUTIONS FOR DYNAMIC DEMAND PROFILES

\begin{tabular}{|c|c|c|c|c|c|c|}
\hline \multicolumn{7}{|c|}{ (EXPERIEMENT 2) } \\
\hline \multicolumn{3}{|c|}{ Experiment 2 setting } & \multicolumn{2}{|c|}{ SNC solutions } & \multicolumn{2}{|c|}{$\%$ Saving } \\
\hline $\begin{array}{c}\text { Cus. } \\
\text { ID }\end{array}$ & $\begin{array}{c}\text { Order } \\
\text { arrival } \\
\text { day }\end{array}$ & $\begin{array}{c}\text { Demand } \\
\text { profile } \\
\left(Q_{l t}, D T_{l t}, P_{l t}\right)\end{array}$ & TNC range & DT range & Cost & Time \\
\hline \multirow[t]{3}{*}{1} & \multirow[t]{3}{*}{1} & $(33,30,1000)$ & {$[717,746]$} & {$[17,21]$} & {$[25,28]$} & {$[30,43]$} \\
\hline & & $(20,15,900)$ & {$[727,852]$} & {$[11,14]$} & {$[5,19]$} & {$[7,27]$} \\
\hline & & $(55,32,1030)$ & {$[704,874]$} & {$[21,29]$} & {$[15,32]$} & {$[9,34]$} \\
\hline \multirow[t]{3}{*}{2} & \multirow[t]{3}{*}{10} & $(40,15,900)$ & {$[710,786]$} & {$[20,23]$} & {$[13,21]$} & $-[33,53]$ \\
\hline & & $(20,15,1030)$ & {$[726,948]$} & {$[12,14]$} & {$[8,30]$} & {$[7,20]$} \\
\hline & & $(45,25,1000)$ & {$[707,820]$} & {$[18,25]$} & {$[18,29]$} & {$[0,28]$} \\
\hline \multirow[t]{3}{*}{3} & \multirow[t]{3}{*}{20} & $(100,55,1300)$ & {$[696,788]$} & {$[38,46]$} & {$[39,46]$} & {$[16,31]$} \\
\hline & & $(80,23,1100)$ & {$[696,1030]$} & {$[24,38]$} & {$[6,37]$} & $-[4,65]$ \\
\hline & & $(150,70,1265)$ & {$[690,1012]$} & {$[41,64]$} & {$[2,45]$} & {$[9,70]$} \\
\hline \multirow[t]{5}{*}{4} & \multirow[t]{5}{*}{45} & $(100,40,1100)$ & {$[699,762]$} & {$[28,34]$} & {$[31,36]$} & {$[15,30]$} \\
\hline & & $(150,40,1000)$ & {$[703,998]$} & {$[44,63]$} & {$[0.2,30]$} & $-[0.4,58]$ \\
\hline & & & {$[1000,1007]$} & {$[41,46]$} & {$[(-0.7), 0]$} & $-[0.1,0.6]$ \\
\hline & & $(152,50,900)$ & {$[704,789]$} & {$[50,63]$} & {$[12,22]$} & {$[-(1.3), 0]$} \\
\hline & & & {$[799,1731]$} & {$[42,50]$} & {$[-92,11]$} & {$[0,16]$} \\
\hline \multirow[t]{3}{*}{10} & \multirow[t]{3}{*}{120} & $(50,40,700)$ & {$[704,737]$} & {$[22,26]$} & $-[0.5,5.2]$ & {$[35,45]$} \\
\hline & & $(45,40,675)$ & {$[704,827]$} & {$[17,24]$} & $-[4,23]$ & {$[40,58]$} \\
\hline & & $(70,35,1000)$ & {$[698,931]$} & {$[24,34]$} & {$[7,30]$} & {$[3,32]$} \\
\hline
\end{tabular}

considered using mean and standard deviation for the selected customer region. The proposed multi-objective optimization model was tested in both experiments to minimize TSNC, as well as DT, while satisfying all the constraints listed in section III. NSGA-II was employed to solve the model. Fig. 5 illustrates the Pareto fronts generated by NSGA-II for a few selected demand profiles. The results obtained in experiment 1 are presented in Table II, which includes TSNC and DT ranges for the Pareto-optimal SNCs for each demand profile. Accordingly, potential savings ranges, which reflect the difference between WTP price and TSNC, are reported. The results from experiment 1 can help decision makers to identify suitable SN configurations in the presence of static demand profiles. An appropriate configuration can be selected from this set based on the desired SN evaluation criteria. Table III shows the results of experiment 2 with corresponding savings for dynamic demand profiles. The results obtained in this analysis can be used to find a strategically robust configuration design which can withstand the effects of volatile markets that are characterized by changing WTP price, lead time and volume, or when faced with other disruptions. Additionally, if a 
solution satisfying the desired criteria is not found, then the experiments can be re-run with amended demand profiles or renegotiated terms of supply.

\section{CONCLUSION}

This study developed a novel multi-objective optimization model to enhance SN performance with respect to TSNC and DT when handling SNC decisions under varying demand profiles. A multi-echelon $\mathrm{SN}$ was considered together with a distributed set of supply entities which are available at each node at different stages of the SC. The model incorporated a bidding mechanism to simulate the competing nature of supply entities and to increase the solution quality and computational efficiency through the application of NSGA-II. We tested the model on a refrigerator $\mathrm{SN}$ case study to demonstrate its efficacy. Experimental results revealed that a number of strategic decisions can be supported by the proposed model, in particular, identifying and evaluating robust $\mathrm{SNs}$ to suit varying demand profiles. We contend that dealing with SNC decisions in the manner outlined in this paper can enhance SC capabilities in terms of capacity to withstand anticipated disruptions, as well as developing contingencies in the case of having to recover from disruptions. In future studies, this model can be further extended by incorporating agent-based modeling to represent a distributed decision-making scenario while accounting for both individual entity behaviors and whole of the SN dynamics. Innovative bidding procedures can also be tested to further enhance the real world behavior and performance of SNs when faced with disruptions.

\section{REFERENCES}

[1] D. Mourtzis, and M. Doukas, "Decentralized manufacturing systems review: challenges and outlook," Logistics Research, vol. 5(3-4), pp. 113-121, 2012.

[2] K. Govindan, M. Fattahi, and E. Keyvanshokooh, "Supply chain network design under uncertainty: A comprehensive review and future research directions," European Journal of Operational Research, vol. 263(1), pp.108-141, 2017.

[3] T. G. Schmitt, S. Kumar, K. E. Stecke, F. W. Glover, and M. A. Ehlen, "Mitigating disruptions in a multi-echelon supply chain using adaptive ordering," Omega, vol. 68, pp. 185-198, 2017.

[4] S. Rezapour, R. Z. Farahani, and M. Pourakbar, "Resilient supply chain network design under competition: A case study," European Journal of Operational Research, vol. 259, no. 3, pp. 1017-1035, 2017.

[5] K. Govindan and M. Fattahi, "Investigating risk and robustness measures for supply chain network design under demand uncertainty: A case study of glass supply chain," International Journal of Production Economics, vol. 183, pp. 680-699, 2017.

[6] E. Revilla and M. J. Saenz, "The impact of risk management on the frequency of supply chain disruptions: A configurational approach," International Journal of Operations \& Production Management, vol. 37, no. 5, pp. 557-576, 2017.

[7] M. Kamalahmadi and M. M. Parast, "A review of the literature on the principles of enterprise and supply chain resilience: Major findings and directions for future research," International Journal of Production Economics, vol. 171, pp. 116-133, 2016.

[8] S. Y. Ponomarov and M. C. Holcomb, "Understanding the concept of supply chain resilience," The international journal of logistics management, vol. 20, no. 1, pp. 124-143, 2009.

[9] S. E. Birkie, P. Trucco, and P. Fernandez Campos, "Effectiveness of resilience capabilities in mitigating disruptions: leveraging on supply chain structural complexity," Supply Chain Management: An International Journal, vol. 22, no. 6, pp. 506-521, 2017.
[10] A. Hasani and A. Khosrojerdi, "Robust global supply chain network design under disruption and uncertainty considering resilience strategies: A parallel memetic algorithm for a real-life case study," Transportation Research Part E: Logistics and Transportation Review, vol. 87, pp. 2052, 2016.

[11] C. Braziotis, M. Bourlakis, H. Rogers, and J. Tannock, "Supply chains and supply networks: distinctions and overlaps," Supply Chain Management: An International Journal, vol. 18(6), pp. 644-652, 2013.

[12] R.H. Ballou, "Unresolved issues in supply chain network design," Information Systems Frontiers, vol. 3(4), pp. 417-426, 2001.

[13] S. A. Melnyk, R. Narasimhan, and H. A. DeCampos, "Supply chain design: issues, challenges, frameworks and solutions," 2014.

[14] S. Piramuthu, "Machine learning for dynamic multi-product supply chain formation," Expert Systems with Applications, vol. 29(4), pp. 985990, 2005.

[15] T. H. Truong and F. Azadivar*, "Optimal design methodologies for configuration of supply chains," International Journal of Production Research, vol. 43, pp. 2217-2236, 2005.

[16] B. Nepal, L. Monplaisir and O. Famuyiwa, "A multi- objective supply chain configuration model for new products," International Journal of Production Research, vol. 49, pp. 7107-7134, 2011.

[17] T.P. Harrison, "Global supply chain design," Information Systems Frontiers, vol. 3(4), pp. 413-416, 2001.

[18] M.J. Meixell, and V. B. Gargeya, "Global supply chain design: A literature review and critique," Transportation Research Part E: Logistics and Transportation Review, vol. 41(6), pp. 531-550, 2005.

[19] N. Azad, H. Davoudpour, G. K. Saharidis, and M. Shiripour, "A new model to mitigating random disruption risks of facility and transportation in supply chain network design," The International Journal of Advanced Manufacturing Technology, vol. 70, no. 9-12, pp. 1757-1774, 2014

[20] U.S.S. Dharmapriya, S.B. Kiridena, and N. Shukla, "A review of supply network configuration literature and decision support tools," Industrial Engineering and Engineering Management (IEEM), pp. 149-153, 2016.

[21] L. A. Moncayo-Martínez and D. Z. Zhang, "Multi-objective ant colony optimisation: A meta-heuristic approach to supply chain design," International Journal of Production Economics, vol. 131, pp. 407-420, 2011

[22] B. Yuce, E. Mastrocinque, A. Lambiase, M. S. Packianather, and D. T. Pham, "A multi-objective supply chain optimisation using enhanced Bees Algorithm with adaptive neighbourhood search and site abandonment strategy," Swarm and Evolutionary Computation, vol. 18, pp. 71-82, 2014.

[23] O. Akanle, and D. Zhang, "Agent-based model for optimising supplychain configurations," International Journal of Production Economics, vol. 115(2), pp. 444-460, 2008.

[24] F. Ameri and C. McArthur, "A multi-agent system for autonomous supply chain configuration," The International Journal of Advanced Manufacturing Technology, vol. 66, pp. 1097-1112, 2013.

[25] N. Shukla and S. Kiridena, "A fuzzy rough sets-based multi-agent analytics framework for dynamic supply chain configuration," International Journal of Production Research, vol. 54(23), pp. 69846996, 2016.

[26] K. Deb, K., A. Pratap, A., S. Agarwal, and T.A.M.T. Meyarivan, "A fast and elitist multiobjective genetic algorithm: NSGA-II," IEEE transactions on evolutionary computation, vol.6(2), pp.182-197, 2002.

[27] Y. Umeda, A. Nonomura, and T. Tomiyama, "Study on life-cycle design for the post mass production paradigm", vol.14, pp.149161,2000 .

[28] H. R. Krikke, J. M. Bloemhof-Ruwaard, and I. N. Van Wassenhove, "Dataset of the refrigerator case: design of closed loop supply chains", 2001.

[29] M. Fleischmann, J.M. Bloemhof-Ruwaard, R. Dekker, E. Van Der Laan, J.A. Van Nunen, and L.N. Van Wassenhove, "Quantitative models for reverse logistics: A review," European Journal of Operational Research vol.103 (1), pp. 1-17,1997. 